\title{
MHD simulation of the three-dimensional structure of the heliospheric current sheet
}

\author{
P. L. Israelevich ${ }^{1}$, T. I. Gombosi ${ }^{2}$, A. I. Ershkovich ${ }^{1}$, K. C. Hansen ${ }^{2}$, C. P. T. Groth ${ }^{2}$, D. L. DeZeeuw ${ }^{2}$, \\ and K. G. Powell ${ }^{3}$ \\ 1 Department of Geophysics and Planetary Sciences, Raymond and Beverly Sackler Faculty of Exact Sciences, \\ Tel Aviv University, Ramat Aviv, 69978 Tel Aviv, Israel \\ 2 Space Physics Research Laboratory, Department of Atmospheric, Oceanic and Space Sciences, University \\ of Michigan, Ann Arbor, MI 48109, USA \\ 3 Department of Aerospace Engineering, University of Michigan, Ann Arbor, MI 48109, USA
}

Received 18 April 2001 / Accepted 15 June 2001

\begin{abstract}
The existence of the radial component of the electric current flowing toward the Sun is revealed in numerical simulation. The total strength of the radial current is $\sim 3 \times 10^{9}$ A. The only way to fulfil the electric current continuity is to close the radial electric current by means of field- aligned currents at the polar region of the Sun. Thus, the surface density of the closure current flowing along the solar surface can be estimated as $\sim 4 \mathrm{~A} / \mathrm{m}$, and the magnetic field produced by this current is $B \sim 5 \times 10^{-6} \mathrm{~T}$, i.e. several percent of the intrinsic magnetic field of the Sun. This seems to mean that any treatment of the solar magnetic field generation should take into account the heliospheric current circuit as well as the currents flowing inside the Sun.
\end{abstract}

Key words. solar wind - Sun: magnetic fields

\section{Introduction}

Solar wind outflow from the Sun causes the magnetic field lines originating from opposite hemispheres to extend radially outward and brings them together near the equatorial plane (e.g. Pneuman \& Kopp 1971). These field lines are of opposite magnetic polarities, and thus the interplanetary magnetic field must change sign abruptly within the narrow layer near the equatorial plane. This implies the presence of a thin sheet of a very high current density. The current circulates around the dipole axis in the same direction as the original current generating the dipole field. It is this heliospheric current sheet that separates fields and plasma flows from different hemispheres. Rotation of the Sun twists the interplanetary magnetic field lines in such a way that they take the shape of Archimedean spiral (Parker 1958). Another effect of the rotation is warping of the current sheet plane into a structure that resembles a ballerinas twirling skirt. Because of this warping, the Earth crosses the current sheet at least twice during each solar rotation (sometimes more than twice, if the current sheet is wavy enough). These current sheet crossings are observed at the Earth as crossings of interplanetary sector boundaries, when the polarity of the magnetic field

Send offprint requests to: P. L. Israelevich,

e-mail: peter@luna.tau.ac.il changes its sign (as observed from the Earth) (Wilcox \& Ness 1965).

Another consequence of solar rotation and magnetic field line twisting was predicted by Alfvén (1981). The spiral form of the magnetic field lines means that there is a significant radial component of the electric current in the sheet, along with the azimuthal component. This point is illustrated by Fig. 1a. Solid lines show the magnetic field lines slightly above the magnetic equator whereas dashed lines correspond to those slightly below the equatorial plane. Obviously, the electric current lines should also be spirals (as shown in Fig. 1a by empty lines), thus $\boldsymbol{j}=\nabla \times \boldsymbol{B} / \mu_{0}$ possesses both radial and azimuthal components. The only way to satisfy the electric current continuity is to close the radial electric current by field-aligned currents at the polar region of the sun. This current closure leads to the three-dimensional heliospheric current system schematically depicted by Alfvén (Fig. 1b). Thus, the heliospheric current system produced by the Sun acts like a unipolar generator.

Since the publication of Alfvén's conceptual model of the heliospheric current system very little work has been done to follow up on this idea. Recently, however, several efforts are under way to model the global threedimensional heliosphere. These efforts are motivated by the space weather and Living with a Star initiatives and 

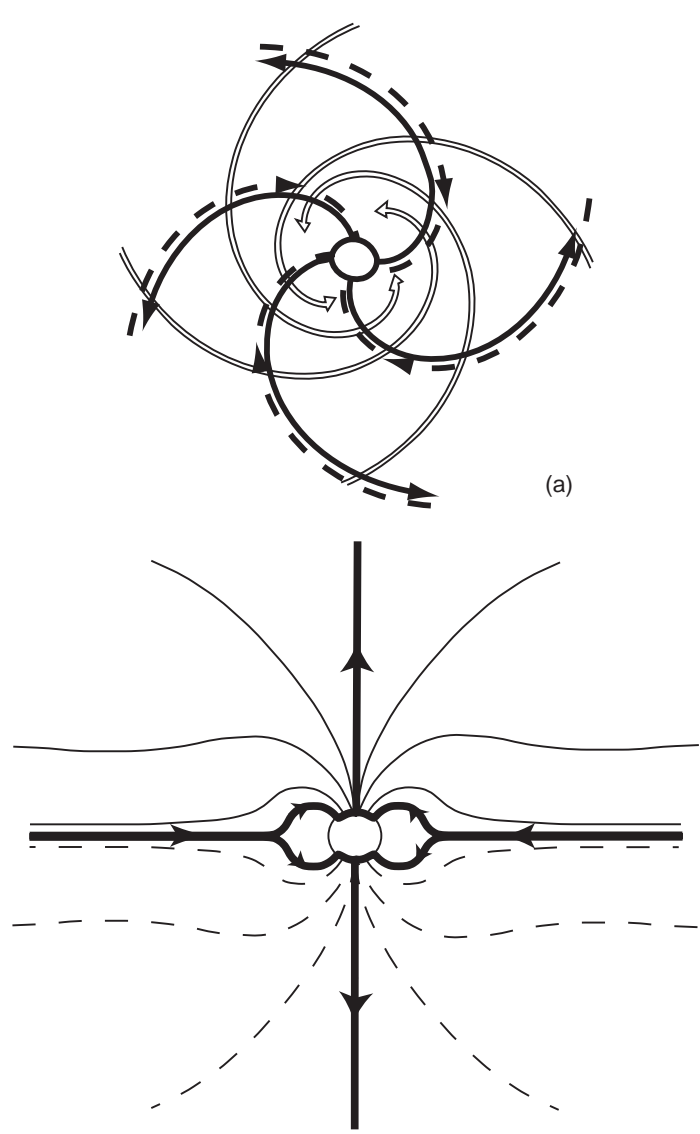

(b)

Fig. 1. a) Interplanetary magnetic field lines and electric current lines in the solar wind near the heliospheric current sheet surface. Solid lines show the magnetic field lines slightly above the surface, and dashed lines correspond to the field lines slightly below the current sheet surface. Empty lines show the direction of the electric current in the sheet. b) Heliospheric current circuit in the meridional plane. Thick lines show the closure of the radial component of the heliospheric electric current.

they, for the first time, attempt the understand the global current system connecting the sun and the heliosphere. The purpose of this note is to show that the three dimensional structure of the heliospheric current system obtained from a self-consistent, first-principles based numerical model of the solar wind outflow with realistic intrinsic solar magnetic field is consistent with Alfvén's conceptual model.

\section{Numerical model}

The work published by Groth et al. (2000) included both a model of the solar corona and solar wind during quiet solar conditions as well as a simulation of a space weather event and its interaction with the Earth. Here we concentrate on the steady state solar wind like solution. The solar wind model is generated by solving the governing equations of ideal MHD using the BATS-R-US code. A detailed description of the code can be found in the papers by Powell et al. (1999). The code employs a high resolution finite volume scheme which is based on an approximate Riemann solver for MHD. In this approach, the single fluid MHD equations are solved for in a fully three-dimensional (no dimensional splitting) tightly coupled manner, rather than in separate steps. BATS-R-US makes use of a block based adaptive grid which allows the model to resolve structures of widely varying length scales.

The computational domain used in the calculation was a rectangular box defined by $-32 R_{\odot} \leq x \leq 224 R_{\odot}$, $-192 R_{\odot} \leq x \leq 192 R_{\odot}$, and $-192 R_{\odot} \leq x \leq 192 R_{\odot}$. The adapted computational grid for the solar wind solution consisted of over $1 \times 10^{6}$ cells with 8 refinement levels. The minimum cell size at the solar surface was $1 / 16 R_{\odot}$, while the cells at $1 \mathrm{AU}$ where approximately $10 R_{\odot}$ in size.

Boundary conditions at the solar surface were dependent on local flow conditions: plasma could freely leave the reservoir, but no "backflow" was allowed. In addition, no deviation was allowed from the intrinsic magnetic field at the surface. Simple zero-gradient boundary conditions are posed at the outer boundaries. The intrinsic solar magnetic field at the solar surface was assumed to be a combination of a dipole and octupole both tilted $15^{\circ}$ from the rotation axis. In order to obtain a nominal solar wind-like solution, Groth et al. (2000) included a volumetric heat source beyond, but near the solar surface. The role of the volumetric heating function is to mimic the combined effects of energy absorption above the transition region, heat conduction (which is not included in ideal MHD) and radiative losses. A complete description of the solar wind model as well as the simulation of the space weather event and its interaction with Earth may be found in Groth et al. (2000).

\section{Results}

Figure 2a shows isolines of $r B_{\varphi}$ (a product of the azimuthal component of the magnetic field by the radial distance) in the meridional plane $X Z$. The thick line $\left(B_{\varphi}=0\right)$ corresponds to the current sheet. It is inclined to the $X$ axis because of inclination of the solar magnetic dipole. The azimuthal field, $B_{\varphi}$, is positive (counterclockwise looking from the north pole) above the current sheet, and negative (clockwise) below it. The fact that the lines of equal $r B_{\varphi}$ approximately can be described as straight lines radiating from the origin of coordinates means that the radial dependence of $B_{\varphi}$ is indeed proportional to $1 / r$ in our simulation as predicted by Parker (1958).

Figure $2 \mathrm{~b}$ shows the isolines of the same quantity, $r B_{\varphi}$ in the equatorial plane $X Y$. The intersection of the current sheet with the equatorial plane is given by the condition $B_{\varphi}=0$, and is shown by the thick line. Note that the isolines of $r B_{\varphi}$ do not necessarily coincide with the magnetic field lines. The quantity $r B_{\varphi}$ equals zero at the surface of the current sheet, and grows with the distance from the surface. Therefore $r B_{\varphi}$-value in the equatorial plane (Fig. 2b) can be considered as a measure of the current sheet distance from the equatorial plane. In other words, it corresponds to the altitude of warps of the current sheet. 
$\mathrm{rB} \varphi$
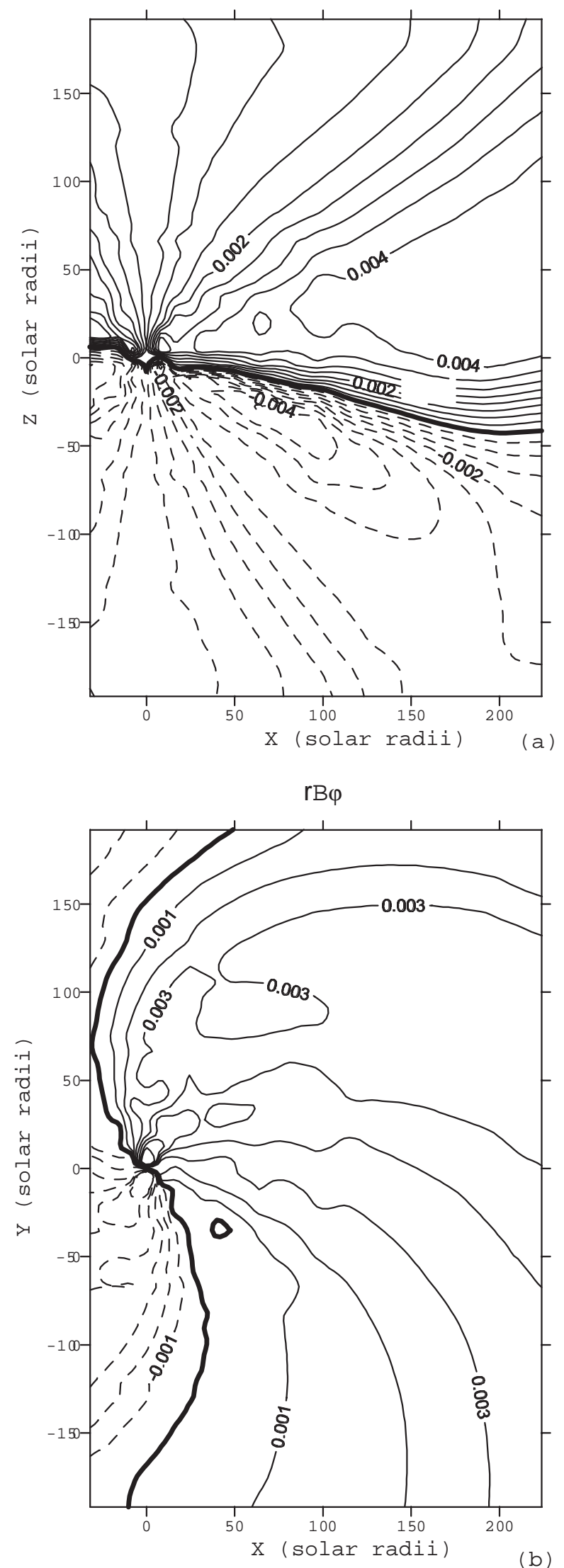

Fig. 2. Isolines of the product of the azimuthal component of the magnetic field $B_{\varphi}$ by the radial distance from the Sun. a) meridional plane and $\mathbf{b}$ ) equatorial plane.
Thus, the current sheet warping clearly exhibits a spiral structure (the ballerina skirt model).

The distributions of the electric current density in the plane perpendicular to the solar magnetic dipole axis $\left(X^{\prime} Y^{\prime}\right)$ are given in Fig. 3. Figure 3a shows isolines of $r j_{\varphi}$, and isolines of $r j_{\mathrm{r}}$ are given in Fig. 3b. The current sheet does not coincide with the plane $X^{\prime} Y^{\prime}$ because of warping. Thus the electric current is stronger at places where the current sheet approaches the $X^{\prime} Y^{\prime}$-plane (this happens in the first and third quadrants in our case). Both radial and azimuthal components of the electric current are present in our simulation. Moreover, since $j_{\varphi}$ decreases with distance much faster than $j_{\mathrm{r}}$, the radial component is dominant at large distances.

Continuity of the electric current requires the radial component closure through the solar atmosphere in a way sketched in Fig. 1b. We are able to follow this closure, and Fig. 4 gives a polar view of isolines of the radial current on the Sun's surface. Indeed, near the magnetic pole the current flows from the Sun. This region of the upward current is surrounded by the downward current. This picture, to some extent, is reminiscent of the regions of field aligned currents in the Earth's polar ionosphere.

In contrast to simulation result (Fig. 4), the positive radial component of the current, according to Alfvén, is limited to a single point (the pole) (see Fig. 1). Such a picture is an idealization which is not confirmed by our numerical simulation. In fact, the results of calculations (presented in Fig. 4) show that there is a continuous transition from positive to negative value of the radial electric current. All the region within the ring of downward (negative) field-aligned current is filled by the positive (upward) current. Unfortunately, more detailed comparison with the Alfvén prediction is impossible since Alfvén (1981) did not discuss this question.

The numerical simulation has been performed for the main solar magnetic field configuration typical for Solar Minimum conditions. During the next Solar Minimum, the solar magnetic dipole polarity is reversed. Obviously, the radial component of the electric current also reverses its sign (Alfvén 1981). Near the Solar Maximum, the dipole component of the main magnetic field almost vanishes. Domination of higher harmonics makes the main field irregular, and, therefore, the regular structure of the heliospheric current system (Fig. 1) disappers. However, radial electric currents should be present in the solar wind current sheets if the plane of the sheet is not parallel to the Sun's rotation axis. The heliospheric magnetic field behavior during the solar cycle can be described as a magnetic flux transport from the equatorial to polar regions of the Sun. During the 11-year solar cycle, the solar wind transfers the magnetic flux equal to the main solar magnetic flux through a hemisphere (Israelevich et al. 2000).

\section{Conclusion}

The three-dimensional heliospheric current system revealed in our simulation may play an important role in 

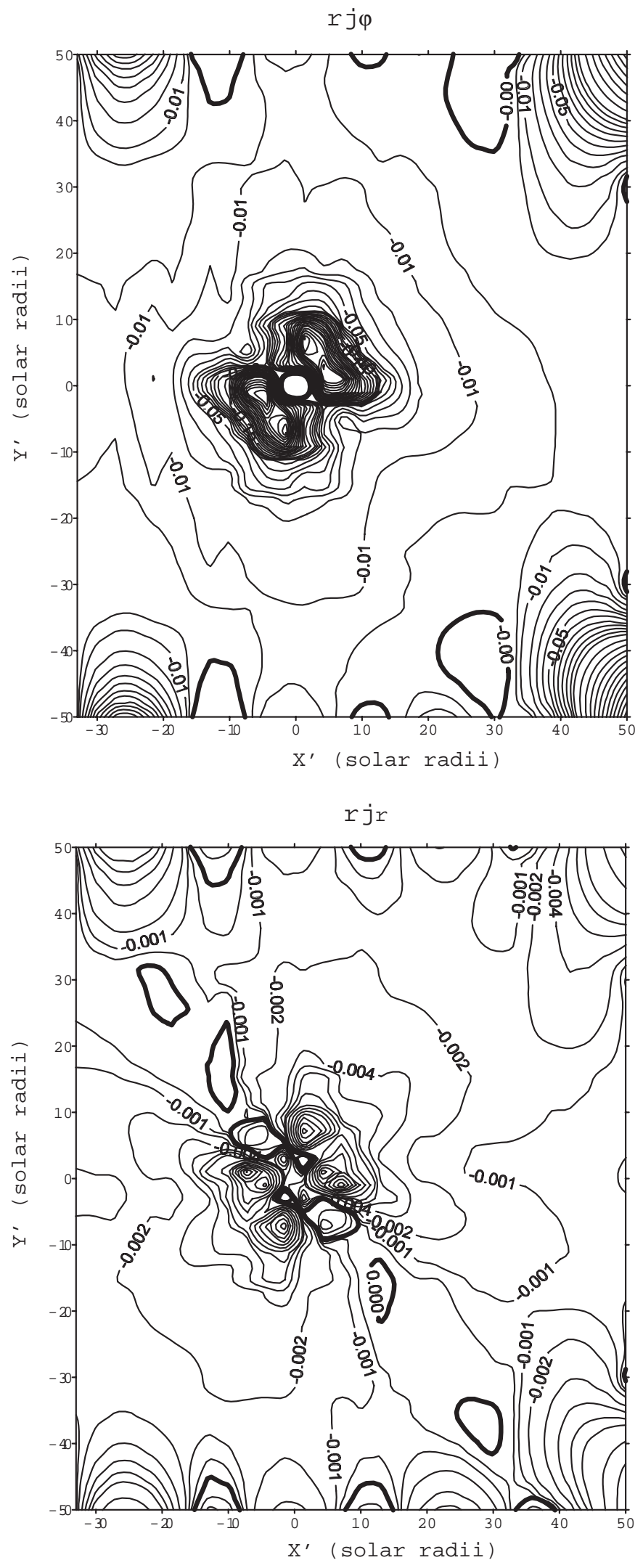

Fig. 3. Isolines of products of the components of the electric current density $j_{\varphi} \mathbf{a}$ ) and $j_{\mathrm{r}} \mathbf{b}$ ) by the radial distance from the Sun shown in the plane perpendicular to the solar magnetic dipole axis.

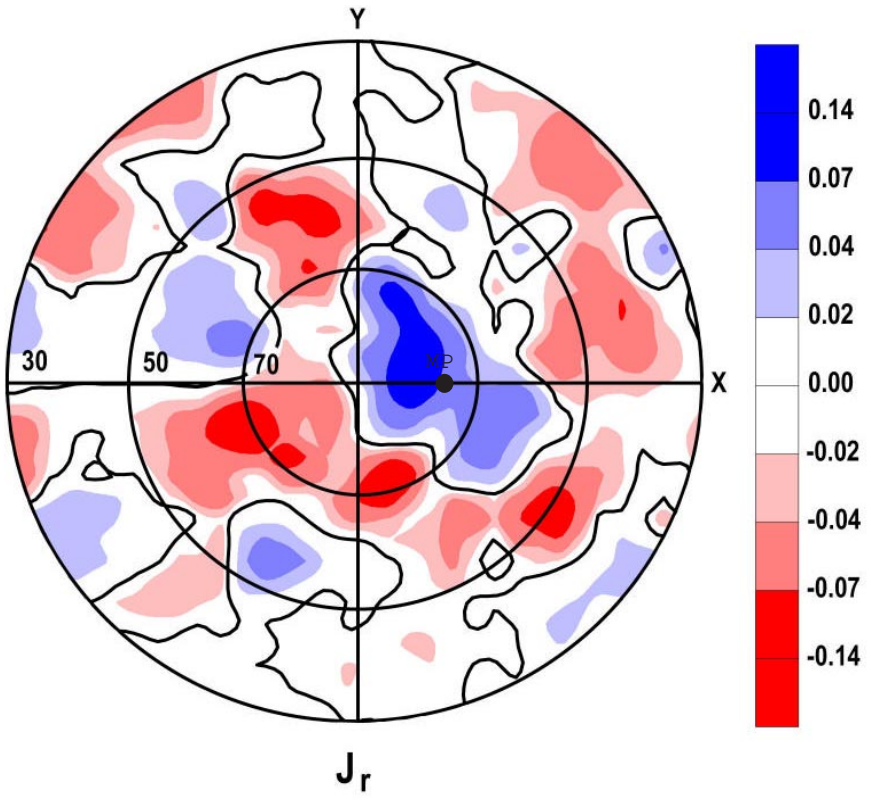

Fig. 4. Polar view of the distribution of the electric current density radial component on the solar surface.

the processes on the Sun. The total strength of the radial current is $\sim 3 \times 10^{9} \mathrm{~A}$. Thus the surface density of the closure current flowing along the solar surface can be estimated as $i=I / R_{\odot} \sim 4 \mathrm{~A} / \mathrm{m}$, and the magnetic field produced by this current is $B=\mu_{0} i \sim 5 \times 10^{-6}$ T, i.e. several percent of the intrinsic magnetic field of the Sun. This seems to mean that any consideration of the solar magnetic field generation should take into account the heliospheric current circuit as well as the currents flowing inside the Sun. Such a conlusion corroborates our recent result (Israelevich et al. 2000) that there is a feedback between the solar wind flow and the main solar magnetic field.

Acknowledgements. This work was supported by the U.S.Israel Binational Science Foundation (BSF).

\section{References}

Alfvén, H. 1981, Cosmic plasma (D. Reidel, Dordrecht) Groth, C. P. T., DeZeeuw, D. L., Gombosi, T. I., \& Powell, K. G. 2000, J. Geophys. Res., 105, 25053

Israelevich, P. L., Ershkovich, A. I., \& Gombosi, T. I. 2000, A\&A, 362, 379

Parker, E. N. 1958, ApJ, 128, 664

Pneuman, G. W., \& Kopp, R. A. 1971, Solar Phys., 18, 258

Powell, K. G., Roe, P. L., Linde, T. J., Gombosi, T. I., \& DeZeeuw, D. L. 1999, J. Comput. Phys., 154, 284

Wilcox, J. M., \& Ness, N. F. 1965, J. Geophys. Res., 70, 5793 